\title{
Pulmonary function impairment of asymptomatic and persistently symptomatic patients 4 months after COVID-19 according to disease severity
}

\author{
Dieter Munker ${ }^{1,2} \cdot$ Tobias Veit $^{1,2} \cdot$ Jürgen Barton ${ }^{1,2} \cdot$ Pontus Mertsch $^{1,2} \cdot$ Carlo Mümmler $^{1,2} \cdot$ Andreas Osterman $^{3,4}$. \\ Elham Khatamzas ${ }^{5}$ Michaela Barnikel ${ }^{1,2}$. Johannes C. Hellmuth ${ }^{5,6} \cdot$ Maximilian Münchhoff $^{3,4,6}$. Julia Walter ${ }^{1,2}$. \\ Alessandro Ghiani ${ }^{7} \cdot$ Stefan Munker ${ }^{8} \cdot J^{\prime}$ lien Dinkel ${ }^{9} \cdot$ Jürgen Behr $^{1,2} \cdot$ Nikolaus Kneidinger $^{1,2} \cdot$ Katrin Milger $^{1,2}$ (I)
}

Received: 10 June 2021 / Accepted: 13 July 2021 / Published online: 28 July 2021

(c) The Author(s) 2021

\begin{abstract}
Objective Evaluation of pulmonary function impairment after COVID-19 in persistently symptomatic and asymptomatic patients of all disease severities and characterisation of risk factors.

Methods Patients with confirmed SARS-CoV-2 infection underwent prospective follow-up with pulmonary function testing and blood gas analysis during steady-state cycle exercise 4 months after acute illness. Pulmonary function impairment (PFI) was defined as reduction below $80 \%$ predicted of DLCOcSB, TLC, FVC, or FEV1. Clinical data were analyzed to identify risk factors for impaired pulmonary function.

Results 76 patients were included, hereof 35 outpatients with mild disease and 41 patients hospitalized due to COVID19. Sixteen patients had critical disease requiring mechanical ventilation, 25 patients had moderate-severe disease. After 4 months, 44 patients reported persisting respiratory symptoms. Significant PFI was prevalent in 40 patients (52.6\%) occurring among all disease severities. The most common cause for PFI was reduced DLCOcSB $(n=39,51.3 \%)$, followed by reduced TLC and FVC. The severity of PFI was significantly associated with mechanical ventilation $(p<0.001)$. Further risk factors for DLCO impairment were COPD $(p<0.001)$, SARS-CoV-2 antibody-Titer $(p=0.014)$ and in hospitalized patients CT score. A decrease of $\mathrm{paO} 2>3 \mathrm{mmHg}$ during cycle exercise occurred in $1 / 5$ of patients after mild disease course. Conclusion We characterized pulmonary function impairment in asymptomatic and persistently symptomatic patients of different severity groups of COVID-19 and identified further risk factors associated with persistently decreased pulmonary function. Remarkably, gas exchange abnormalities were revealed upon cycle exercise in some patients with mild disease courses and no preexisting pulmonary condition.
\end{abstract}

Keywords Post-COVID $\cdot$ Pulmonary function impairment $\cdot$ COVID-19 $\cdot$ SARS-CoV-2

\section{Introduction}

The coronavirus disease 2019 (COVID-19) pandemic has put considerable strain on the health systems globally [1]. A significant percentage of patients develop COVID-19 pneumonia, which is the key determinant of prognosis in COVID-19 [2-4]. Risk factors for critical disease are mainly age, cardiovascular disease, diabetes and male sex [5].

Dieter Munker and Tobias Veit have contributed equally.

Katrin Milger

Katrin.Milger@med.uni-muenchen.de

Extended author information available on the last page of the article
Further pulmonary worsening is mediated by a cascade of hyperinflammatory responses, which is frequently observed after an initial period of symptom stability. COVID-19 pneumonia and the severe inflammatory process are hypothesized to cause lasting parenchymal damage. Besides, patients with moderate to severe COVID-19 disease are more likely to develop multi-organ disease, e.g. myocardial injury, thromboembolic complications, liver failure, and pneumothorax [6-10]. Comparably, patients after Acute Respiratory Distress Syndrome (ARDS) suffer from long-term physical problems and lung function deterioration [6].

An unknown proportion of patients may develop subclinical COVID-19 pneumonia with a mild disease course without hospitalization. So far, few studies have analyzed 
the impact of COVID-19 on lung function, and its long-term effects remain unclear. It is essential to identify risk factors for lasting pulmonary damage and define patients at risk who should receive specialized respiratory follow-up care after infection.

Here, we evaluate pulmonary function impairment after COVID-19 disease among all severity groups in persistently symptomatic and asymptomatic patients four months after infection. Furthermore, this study investigates predictors for lung function impairment according to disease severity in a highly diverse COVID-19 patient group, including ergometry assessment in a pulmonary aftercare setting.

\section{Methods}

\section{Design and study population}

This single-center cohort study included 76 patients with confirmed COVID-19 disease with acute illness during the first wave (March to August 2020). The Regional Ethics Committee approved the study of Ludwig-MaximilianUniversity (LMU) of Munich (project number 20-454), Germany. Up to December 2020, we prospectively included all adult patients (age $>18$ years) who presented to the LMU outpatient department for follow-up 4 months after confirmed SARS-CoV-2 infection [positive polymerase chain reaction (PCR) or positive Anti-SARS-CoV-2 Immunoglobulin $\mathrm{G}(\mathrm{IgG})$ titer]. Hospitalized patients from LMU university hospital were referred to the outpatient clinic after discharge. Additionally, non-hospitalized (mild) cases were referred by the general practitioner. All patients provided written informed consent. Patients underwent a standardized interview to qualitatively evaluate COVID-19 related symptoms (yes or no) as previously described [11]. We differentiated between any COVID-19-related symptoms (including dyspnea, cough, fatigue, or anosmia, yes or no) and specific respiratory symptoms (dyspnea at rest or after exercise, cough, yes or no).

\section{Pulmonary function testing and cycle exercise blood gas analysis}

Pulmonary function testing (PFT) was performed by plethysmography (Masterscreen Body, Jäger), and the following values were analyzed: Total lung capacity in liter (TLC in 1 and \% predicted value), forced vital capacity (FVC in 1 and $\%$ predicted value), forced expiratory volume of $1 \mathrm{~s}$ (in 1), Tiffeneau Index (FEV1/FVC), diffusion capacity of carbon monoxide by single breath test in $\mathrm{mmol} / \mathrm{minute} /$ kiloPascal (DLCOcSB in $\mathrm{mmol} / \mathrm{min} / \mathrm{kPa}$ and $\%$ predicted value, corrected for hemoglobin). For plethysmographic ECSC [12] normal values were used, for spirometry GLI
2012 [13] normal values were used. For the definition of obstruction lower limit of normal (LLN) according to GLI 2012 was used (2).

If bronchial hyperresponsiveness was clinically suspected, inhaled methacholine testing was performed stepwise (Dosage: $0.05 \mathrm{mg}, 0.15 \mathrm{mg}, 0.45 \mathrm{mg}$ ). Bronchial hyperresponsiveness was diagnosed if FEV1 declined by $>20 \%$ at a methacholine concentration $<=4 \mathrm{mg} / \mathrm{ml}$ $\left(\mathrm{PC}_{20}\right.$ (provocative concentration causing a $20 \%$ fall in FEV1) [14].

Also, if bronchial obstruction (Tiffeneau Index $<70 \%$ ) was detected, bronchodilator reversibility was tested using two puffs (100 mg/puff) of salbutamol [14].

Arterialized capillary blood gas analysis from the earlobe was performed at rest and after cycle ergometry. We preferred sampling from ear lobe capillaries in an outpatient setting due to the lower risk of complications including aneurysms, fistulas, ischemia infection, and hematoma. In the absence of contraindications, eligible patients performed steady-state cycle ergometry with stable resistance of $2 \mathrm{~W} /$ $\mathrm{kg}$ (ideal body weight) over 6 min with blood gas analysis performed during steady-state exercise at $5 \mathrm{~min}$ time point. Contraindications were severe resting hypoxia requiring long-term oxygen therapy, other medical conditions such as myocarditis, and incapacity to cycle due to orthopedic problems. As RQ was not directly measured, for calculation of $\mathrm{AaDO} 2$ under exercise, following simplified formula was used: $\mathrm{AaDO} 2=140$ (altitude Munich $530 \mathrm{~m}$ ) - paO2 - paCO2.

\section{Diagnosis of COVID 19}

We diagnosed COVID-19 in patients with matching symptoms and documented positivity for SARS-CoV-2 RNA in a reverse transcriptase-polymerase chain reaction (RT-PCR) from nasopharyngeal swabs (NPS) as described previously [15]. In case of high clinical suspicion but negativity for SARS-CoV-2 RNA in available clinical samples, e.g. false negatives due to limited quality of clinical sampling, absence of a PCR test due to low testing capacity at the beginning of the COVID-19 pandemic, we assumed a previous infection with SARS-CoV-2 after detection of specific antibodies against SARS-CoV-2.

\section{Anti-SARS-CoV-2 IgG detection}

Serum samples were measured using Anti-SARS-CoV-2IgG-ELISA (Euroimmun AG, Lübeck, Germany) at the time of presentation for PFTs. The assay was performed according to the manufacturer's instructions. Borderline results were considered positive in this study. 


\section{Disease severity and comorbidity}

Patients were divided into 3 groups (mild, moderate/severe, and critical severity) according to WHO criteria (https:// www.who.int/publications/i/item/clinical-management-ofcovid-19). Moderate and severe disease courses were analysed as one group (moderate/severe). Symptomatic patients without hospitalization were classified as mild disease. Hospitalized patients with clinical signs of pneumonia without oxygen requirement were classified as moderate disease and patients who needed low-flow oxygen supplementation via nasal cannula were classified as severe disease. Critically ill patients received non-invasive ventilation (NIV) or invasive mechanical ventilation. Charlson Comorbidity Index was used to classify degree of comorbidity [16].

\section{Chest $\mathrm{CT}$ protocols and image analysis}

If performed initially, CT scans were included in the analysis. All CT scans were performed at the end of inhalation using 64 row CT scanner with a detector configuration of $64 \times 0.6 \mathrm{~mm}$ or using a 16 -row CT scanner.

One radiologist and one pulmonologist screened the CT scans according to the radiologic scoring system from 0 to 25 points, which has been previously used to describe the extent of ground-glass opacities in SARS-CoV-2 infection [17-19].

\section{Nasopharyngeal swabs (NPS) and viral load analysis}

NPS were routinely obtained on admission and according to local guidelines. NPS samples were taken on clinical suspicion of COVID-19. At admission, up to two NPS samples (with at least $12 \mathrm{~h}$ distance) and, if necessary, one sputum sample was obtained.

Repeated collection of either sample (NPS, sputum, and endotracheal aspirates) was performed in hospitalized patients for clinical monitoring purposes. When COVID19 symptoms resolved and two consecutive NPS (at least with a day distance) showed a negative result, testing was stopped [15]. In this analysis, we used the term "duration of positivity for viral shedding." It expresses the duration in days between the first and last confirmed positivity for SARS-CoV-2 in days. Different formulae were derived for each PCR assay to convert $C t / C p$ values to copy number estimates as described previously $[15,20]$.

\section{Statistical analysis}

Continuous variables were reported using mean with standard deviation (SD). We compared differences in means using unpaired $t$-test and ANOVA in the whole sample. When comparing repeated measurements of lung function testing, we used paired $t$ test and Mann-Whitney test. Categorical variables were reported as absolute and relative frequencies and compared using $\chi^{2}$ test. In the univariate analysis we used Pearson correlation coefficient to test for association between variables. In the multivariate analysis, we applied multivariate linear regression to study the association of different covariates (e.g. SARS-CoV-2 IgG, Charlson Comorbidity Index, COPD, need for pulmonary ventilation) on changes in pulmonary function parameters (DLCOcSB, TLC, FVC, FEV1). Statistical significance was considered at $p<0.05$. SPSS Version 26 and GraphPad Prism Version 9 were used for statistical analysis.

For descriptive purposes, pulmonary function impairment (PFI) was defined as a decrease of $<80 \%$ of predicted in any of the following: DLCOcSB, TLC, FVC, or FEV1.

\section{Results}

\section{Baseline characteristics}

Up to December 2020, 76 patients were included. The mean age $( \pm \mathrm{SD})$ was $49.6 \pm 17.4$, and $43.3 \%$ of the patients were male. Thirty-five patients $(46.1 \%)$ had mild disease treated as outpatients. In these mild patients, no imaging studies were performed during acute illness as patients were quarantined. 41 patients (53.9\%) were hospitalized and had CT scan during acute illness. Hereof 25 patients (32.9\%) had moderate/severe disease while 16 patients $(21.1 \%)$ developed critical disease during hospitalization with the necessity for mechanical ventilation (Table 1). Patients with mild and moderate/severe disease were significantly younger than patients with critical disease with mean age $( \pm$ SD) $44.3 \pm 14.6,48.1 \pm 19.6$ and $63.8 \pm 10.3$ years, respectively). 10 patients (24\%) who were scheduled for appointments were lost to follow-up (Fig. 1).

The majority of patients $(n=53,69.7 \%)$ reported at least one persistent COVID-19 related symptom at presentation in our center four months after the initial diagnosis of COVID19. Persistent respiratory symptoms (dyspnea at rest, dyspnea after exercise, or cough) were documented in 43 (56.6\%) patients. 21 patients $(27.6 \%)$ reported persistent fatigue, and 12 patients (15.8\%) persistent anosmia (Table 2). One-third (24 patients) reported no residual symptoms at the follow-up presentation.

Comorbidities were frequent, with an average Charlson comorbidity score of $1.3 \pm 1.8$, with arterial hypertension $(22.4 \%)$ and obesity (17\%) being most common. Fourteen patients $(18.4 \%)$ had a previously known chronic lung disease (COPD $n=5,6.6 \%$; bronchial asthma $n=9,11.8 \%$, Table 1). According to patient interview, no patient had been diagnosed with interstitial lung disease previously. Nine patients were immunocompromised $(n=3$ with hematologic 
Table 1 Baseline characteristics of the study population

\begin{tabular}{|c|c|c|c|c|c|}
\hline & Total cohort & $\begin{array}{l}\text { Mild } \\
\text { No hospitalization }\end{array}$ & $\begin{array}{l}\text { Moderate/severe disease } \\
\text { Hospitalization, } \\
\text { No mechanical ventilation }\end{array}$ & $\begin{array}{l}\text { Critical disease } \\
\text { Mechanical ventilation } \\
\text { (non-invasive or intubation } \\
\text { necessary) }\end{array}$ & $p$ value \\
\hline & $n=76$ & $n=35$ & $n=25$ & $n=16$ & \\
\hline Age, mean $\pm \mathrm{SD}$ & $49.6 \pm 17.4$ & $44.3 \pm 14.6$ & $48.1 \pm 19.6$ & $63.8 \pm 10.3$ & $<0.001 *$ \\
\hline Male, $n(\%)$ & $33(43.4)$ & $13(37.1)$ & $9(36.0)$ & $12(70.6)$ & 0.285 \\
\hline Oxygen insufflation, $n(\%)$ & $n=28(36.8)$ & n.a. & $n=12(48.0 \%)$ & $n=16(100.0 \%)$ & 0.389 \\
\hline $\begin{array}{l}\text { mechanical ventilation } \\
\text { (days) } \pm \mathrm{SD}\end{array}$ & n.a. & n.a. & n.a. & $19.1 \pm 15.3$ & n.a. \\
\hline $\begin{array}{l}\text { Days of hospitaliza- } \\
\text { tion } \pm \text { SD }\end{array}$ & n.a. & n.a. & $8.7 \pm 5.9$ & $39.2 \pm 34.6$ & $0.015 *$ \\
\hline $\begin{array}{l}\text { Thoracic CT available in } n \\
\text { (\%) patients }\end{array}$ & $45(59.2 \%)$ & n.a. & $19(76 \%)$ & $16(100 \%)$ & 0.489 \\
\hline CT score (range $0-25^{*}$ ) & n.a. & n.a. & $5.8 \pm 4.7$ & $12.0 \pm 7.4$ & $<0.001 *$ \\
\hline $\begin{array}{l}\text { Peak viral load in nose } \\
\text { swabs }(\text { copies } / \mathrm{ml}) \pm \mathrm{SD}\end{array}$ & $151.9 \times 10^{6} \pm 623.8 \times 10^{6}$ & n.a. & $45.4 \times 10^{6} \pm 125.4 \times 10^{6}$ & $310.7 \pm 910.1 \times 10^{6}$ & 0.337 \\
\hline $\begin{array}{l}\text { Duration of viral shedding } \\
\text { in days (last confirmed } \\
\text { positivity) } \pm \text { SD }\end{array}$ & $17.6 \pm 15.8$ & n.a. & $16.0 \pm 14.8$ & $19.9 \pm 17.3$ & 0.131 \\
\hline $\begin{array}{l}\text { Charlson Comorbidity } \\
\text { Index } \pm \mathrm{SD}, n(\%)\end{array}$ & $1.3 \pm 1.8$ & $0.6 \pm 1.3$ & $1.7 \pm 2.0$ & $2.0 \pm 1.9$ & $0.007 *$ \\
\hline COPD, $n(\%)$ & $5(6.6 \%)$ & $3(8.6 \%)$ & $1(4.0 \%)$ & $1(6.3 \%)$ & 0.136 \\
\hline Bronchial asthma, $n(\%)$ & $9(11.8 \%)$ & $5(14.3 \%)$ & $2(8.0 \%)$ & $2(12.5 \%)$ & 0.073 \\
\hline History of smoking, $n(\%)$ & $12(15.8 \%)$ & $5(14.3 \%)$ & $3(12.0 \%)$ & $4(25.0 \%)$ & 0.627 \\
\hline Arterial hypertension $n(\%)$ & $17(22.4 \%)$ & $3(8.6 \%)$ & $7(28.0 \%)$ & $7(43.8 \%)$ & 0.071 \\
\hline $\begin{array}{l}\text { Diabetes mellitus type } 2, \\
n(\%)\end{array}$ & $4(5.3 \%)$ & $0(0 \%)$ & $2(8.0 \%)$ & $2(12.5 \%)$ & 0.553 \\
\hline $\begin{array}{l}\text { Coronary artery disease, } \\
n(\%)\end{array}$ & $5(7.6 \%)$ & $1(2.9 \%)$ & $2(8.0 \%)$ & $2(12.5 \%)$ & 0.462 \\
\hline Obesity, $n(\%)$ & $8(13.6 \%)$ & $1(2.9 \%)$ & $3(12.0 \%)$ & $5(31.3 \%)$ & $0.045^{*}$ \\
\hline $\begin{array}{l}\text { Obstructive sleep apnea, } \\
n(\%)\end{array}$ & $1(1.3 \%)$ & 0 & 0 & $1(6.3 \%)$ & 0.39 \\
\hline Immunosuppression $*, n(\%)$ & $9(12.1 \%)$ & $2(5.7 \%)$ & $3(12.0 \%)$ & $4(25.0 \%)$ & 0.232 \\
\hline
\end{tabular}

Data are mean (SD) or $n(\%)$. $p$ values were calculated by ANOVA, Mann-Whitney $U$ test, or $\chi^{2}$ test, as appropriate. COVID-19 typical changes included either ground-glass opacities or diffuse bilateral infiltrates. Duration of nasopharyngeal viral shedding was defined by the time between symptom begin and last positivity for viral shedding in standardized nose swabs

*Defined as immunodeficiency due to primary or secondary hematologic disease $n=3(3.9 \%)$ or current immunosuppressant medication due to rheumatic disease $n=6(7.9 \%)$

p-values $<0.05$ were considered significant and marked in bold

disorders, $n=6$ under immunosuppressants due to rheumatic disease).

\section{Pulmonary function testing at 4-month follow-up}

DLCOcSB was significantly lower after critical COVID-19 disease $(60.6 \pm 19.5 \%$ of predicted $)$ compared to moderate/severe $(81.4 \pm 11.9 \%$ of predicted $)$ and mild disease $(79.9 \pm 15.5 \%$ of predicted) courses ( $p<0.01$, Table 2 and Fig. 2A). Likewise, TLC, FVC, and FEV1 were significantly decreased after critical disease course, while mean FEV1/FVC was normal in all disease severities (Table 2 and Fig. 2A). Resting arterialized capillary partial oxygen pressure (paO2) was significantly lower $(65.3 \pm 10.2 \mathrm{mmHg})$ in patients after critical disease $(p<0.01)$ compared to moderate/severe $(76.2 \pm 8.0 \mathrm{mmHg})$ and mild disease $(78.7 \pm 6.6 \mathrm{mmHg})$ (Table 2 and Fig. 2B). Mean paCO2 was within normal range $(36.2 \pm 3.6 \mathrm{mmHg})$ and did not differ between the severity groups $(p=0.37)$. During cycle ergometry, blood gas analysis (BGA) was performed in 43 eligible patients (56.6\%). During ergometry, $\mathrm{paO} 2$ decreased $>3 \mathrm{mmHg}$ in 13 patients (30\%). A paO2 decrease was noted in 3 out of 8 patients (38\%) after critical COVID 19 disease, in 5 out of 


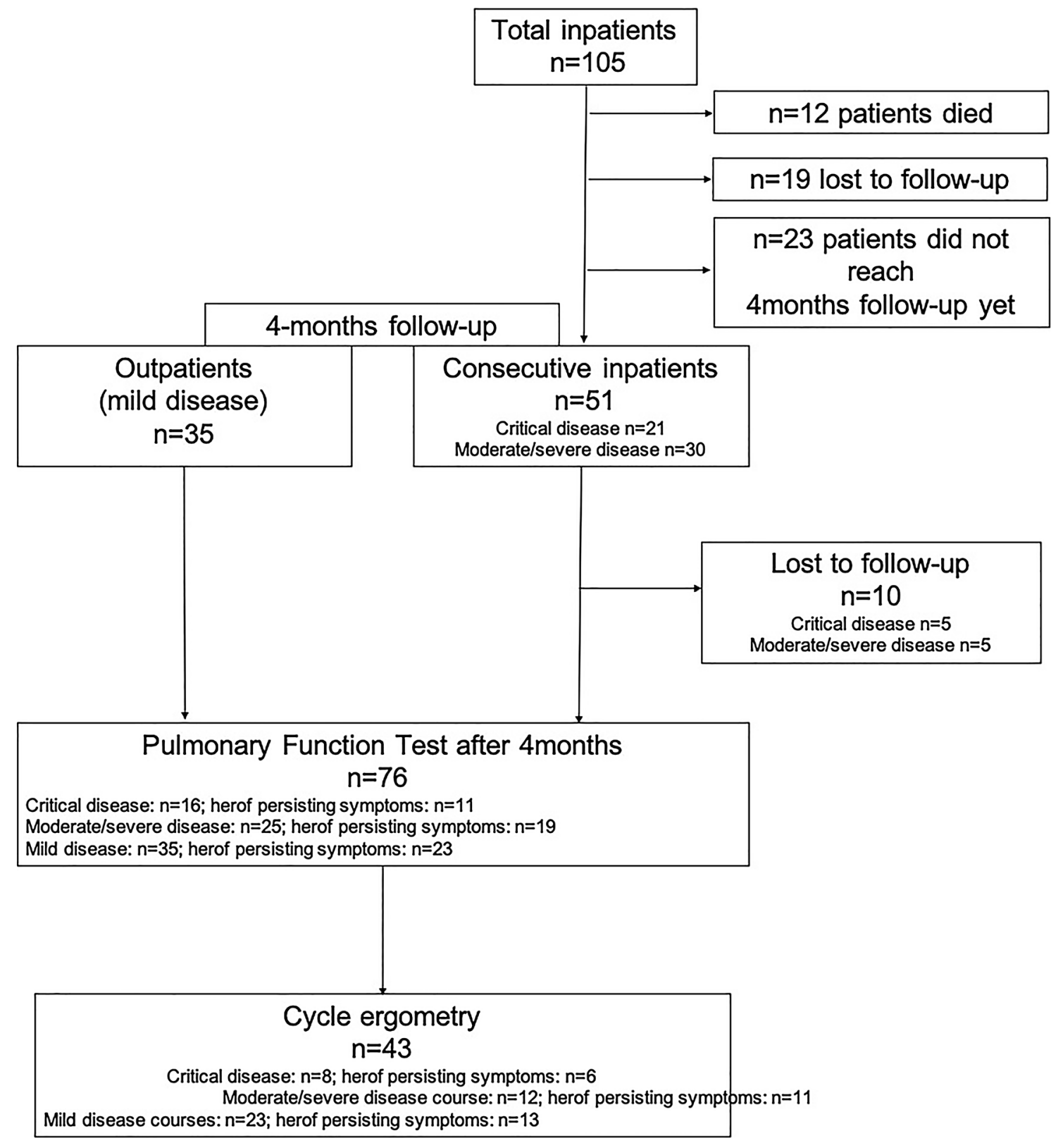

Fig. 1 Study overview. Persisting symptoms: at least one symptom reported at 4 month-follow-up

12 patients (42\%) moderate/severe disease, and 5 out of 23 patients (22\%) with mild disease (Fig. 2B). AaDO2 under exercise was significantly higher in critical patients compared to the other groups, but using a cutoff of $25 \mathrm{mmHg}$ abnormal values were also observed in patients after mild disease (Fig. 2B).

If FEV1/FVC indicated obstructive ventilatory defect $(<$ LLN), bronchodilator reversibility with salbutamol was tested $(n=10)$. Reversibility was confirmed in one $(10 \%)$ patient. Additionally, 8 patients with normal lung function but persisting cough or dyspnea were challenged with inhaled methacholine. Bronchial hyperresponsiveness was newly diagnosed in 3 patients after SARS-CoV-2 infection.

\section{Risk factors for pulmonary function impairment}

Next, we investigated risk factors for pulmonary function impairment using univariate regression analysis. Here, mechanical ventilation, duration of mechanical ventilation, duration of hospitalization, oxygen insufflation, Charlson Index, COPD, smoking history, obesity and 
Table 2 Pulmonary function testing and blood gas analysis

\begin{tabular}{|c|c|c|c|c|c|}
\hline & All patients & $\begin{array}{l}\text { Mild disease } \\
\text { Outpatients } \\
\text { No hospitalization }\end{array}$ & $\begin{array}{l}\text { Moderate/ } \\
\text { severe disease } \\
\text { Hospitaliza- } \\
\text { tion, } \\
\text { No mechanical } \\
\text { ventilation }\end{array}$ & $\begin{array}{l}\text { Severe disease } \\
\text { Mechanical ventilation (non- } \\
\text { invasive or intubation necessary) }\end{array}$ & $p$ value \\
\hline Patients, $n$ & $n=76$ & $n=35$ & $n=25$ & $n=16$ & \\
\hline Persistent COVID-19-related symptoms* & $53(69.7 \%)$ & $23(65.7 \%)$ & $19(76.0 \%)$ & $11(68.8 \%)$ & 0.435 \\
\hline $\begin{array}{l}\text { Persistent COVID-19-related respiratory } \\
\text { symptoms** }\end{array}$ & $43(56.6 \%)$ & $15(42.9 \%)$ & $17(68.0 \%)$ & $11(68.8 \%)$ & 0.233 \\
\hline \multicolumn{6}{|l|}{ Spirometry } \\
\hline TLC, L & $5.8 \pm 1.5$ & $6.1 \pm 1.3$ & $5.9 \pm 1.6$ & $5.1 \pm 1.7$ & 0.11 \\
\hline TLC, $\%$ of predicted & $96.3 \pm 19.1$ & $99.8 \pm 11.4$ & $101.8 \pm 21.4$ & $77.5 \pm 19.8$ & $<0.01 *$ \\
\hline FVC, L & $3.7 \pm 1.1$ & $4.0 \pm 1.1$ & $3.7 \pm 0.8$ & $2.9 \pm 1.1$ & $<0.01 *$ \\
\hline $\mathrm{FVC}, \%$ of predicted & $97.1 \pm 17.6$ & $99.4 \pm 13.9$ & $102.5 \pm 16.1$ & $79.7 \pm 20.0$ & $<0.01 *$ \\
\hline FEV1, L & $3.0 \pm 0.9$ & $3.3 \pm 0.9$ & $3.1 \pm 0.8$ & $2.4 \pm 0.9$ & $<0.01 *$ \\
\hline FEV $1, \%$ of predicted & $97.0 \pm 18.5$ & $97.1 \pm 14.5$ & $104.3 \pm 16.5$ & $84.1 \pm 23.5$ & $<0.01 *$ \\
\hline FEV1/FVC & $83.5 \pm 7.5$ & $82.4 \pm 7.3$ & $84.6 \pm 4.8$ & $84.5 \pm 23.5$ & 0.46 \\
\hline \multicolumn{6}{|l|}{ Blood gases and diffusion } \\
\hline DLCOcSB $(\mathrm{mmol} / \mathrm{min} / \mathrm{kPa})$ & $7.2 \pm 2.2$ & $7.9 \pm 2.2$ & $7.4 \pm 1.5$ & $5.5 \pm 2.2$ & $<0.01 *$ \\
\hline DLCOcSB, $\%$ of predicted & $77.2 \pm 16.5$ & $79.9 \pm 15.5$ & $81.4 \pm 11.9$ & $61.2 \pm 18.0$ & $<0.01 *$ \\
\hline \multicolumn{6}{|l|}{ At rest } \\
\hline $\mathrm{paO} 2(\mathrm{mmHg})$ & $75.5 \pm 9.2$ & $78.7 \pm 6.6$ & $76.2 \pm 8.0$ & $67.3 \pm 11.0$ & $<0.01 *$ \\
\hline $\mathrm{paCO} 2(\mathrm{mmHg})$ & $36.2 \pm 3.6$ & $36.2 \pm 3.6$ & $35.4 \pm 3.2$ & $37.3 \pm 4.2$ & 0.94 \\
\hline
\end{tabular}

Data are mean (SD) or $n(\%) . p$ values were calculated by Mann-Whitney $U$ test or $\chi^{2}$ test, as appropriate

$T L C$ total lung capacity, FVC forced vital capacity, FEVI forced expiratory volume in one second, Tiffeneau Index (FEV1/FVC) DLCOcSB diffusing capacity of the lung for carbon monoxide single-breath corrected for hemoglobin, $\mathrm{pO} 2$ capillary partial oxygen pressure, $\mathrm{pCO} 2$ capillary partial carbon dioxide pressure

*Dyspnea, cough, fatigue, or anosmia

$\mathrm{p}$-values $<0.05$ were considered significant and marked in bold

SARS-CoV2-IgG antibody titer were significantly correlated with pulmonary function parameters (Table 3a).

Using multivariate regression analysis (MLR), the strongest risk factor for persistent pulmonary function impairment in the total cohort $(n=76)$ was mechanical ventilation (DLCOcSB: $p=0.009^{*}$, TLC: $p<0.001^{*}$, FVC: $p<0.001$, FEV1: $p=0.001$; Table $3 b$ ). Further, COPD was associated with reduced DLCO, TLC, FVC, FEV1 after correcting for influences by other variables in multivariate regression analysis and SARS-CoV-2 IgG titer at 4 months was negatively correlated with DLCO among all subgroups (MLR: $p=0.014$, $p=0.042$, Table 3b). Looking at the inpatients $(n=41)$ with CT scans available during hospital stay, MLR revealed additional negative correlations between DLCO at 4 months and CT scores during hospital stay ( $p=0.026$ ) (Table $3 c$ ). In noncritical patients (mild + moderate/severe: $n=60$ ) there was no correlation of oxygen insufflation and length of hospitalization to pulmonary function parameters, whereas preexisting immunosuppression was associated with reduced DLCO (Table $3 \mathrm{~d}$ ).

\section{Pulmonary function impairment (PFI) in persistently symptomatic versus non-symptomatic post-COVID-19 patients}

Significant pulmonary function impairment (PFI) defined as any value $<80 \%$ predicted, was detected in $40(52.6 \%)$ patients. As there was a trend for an association of respiratory symptoms and reduced pulmonary function parameters in univariate regression analysis (Table $3 \mathrm{a}$ ), we further investigated a possible association of symptoms and PFI. No significant difference appeared in lung function parameters when comparing asymptomatic and patients with persisting COVID-19-related symptoms (Figure S1). Likewise, looking only at patients with mild disease courses, symptomatic and asymptomatic patients had no significant differences in lung function parameters (DLCO: $p=0.267$; TLC: $p=0.814$, FVC: $p=0.823$, FEV1: $p=0.991$ ), and proportions of patients with impaired pulmonary function were similar among persistently symptomatic and asymptomatic patients (Table S1). Within the mild disease group, persistently symptomatic (all COVID-19-related symptoms) patients 
Fig. 2 A Pulmonary function testing 4 months after acute illness in patients with mild, moderate/severe and critical disease course. Mean and individual values are displayed, all parameters are shown as \% predicted. DLCOcSB diffusion capacity for CO Single breath, FVC Forced vital capacity,

TLC Total lung capacity, FEVI Forced Expiratory Volume in $1 \mathrm{~s}, R V$ Residual Volume. Statistical testing performed by ANOVA + Tukey's multiple comparison test. $\mathbf{B}$ paO2 and AaDO2 from arterialized capillary blood gas analysis at rest and during at exercise according to COVID-19 disease severity

4 months after acute illness
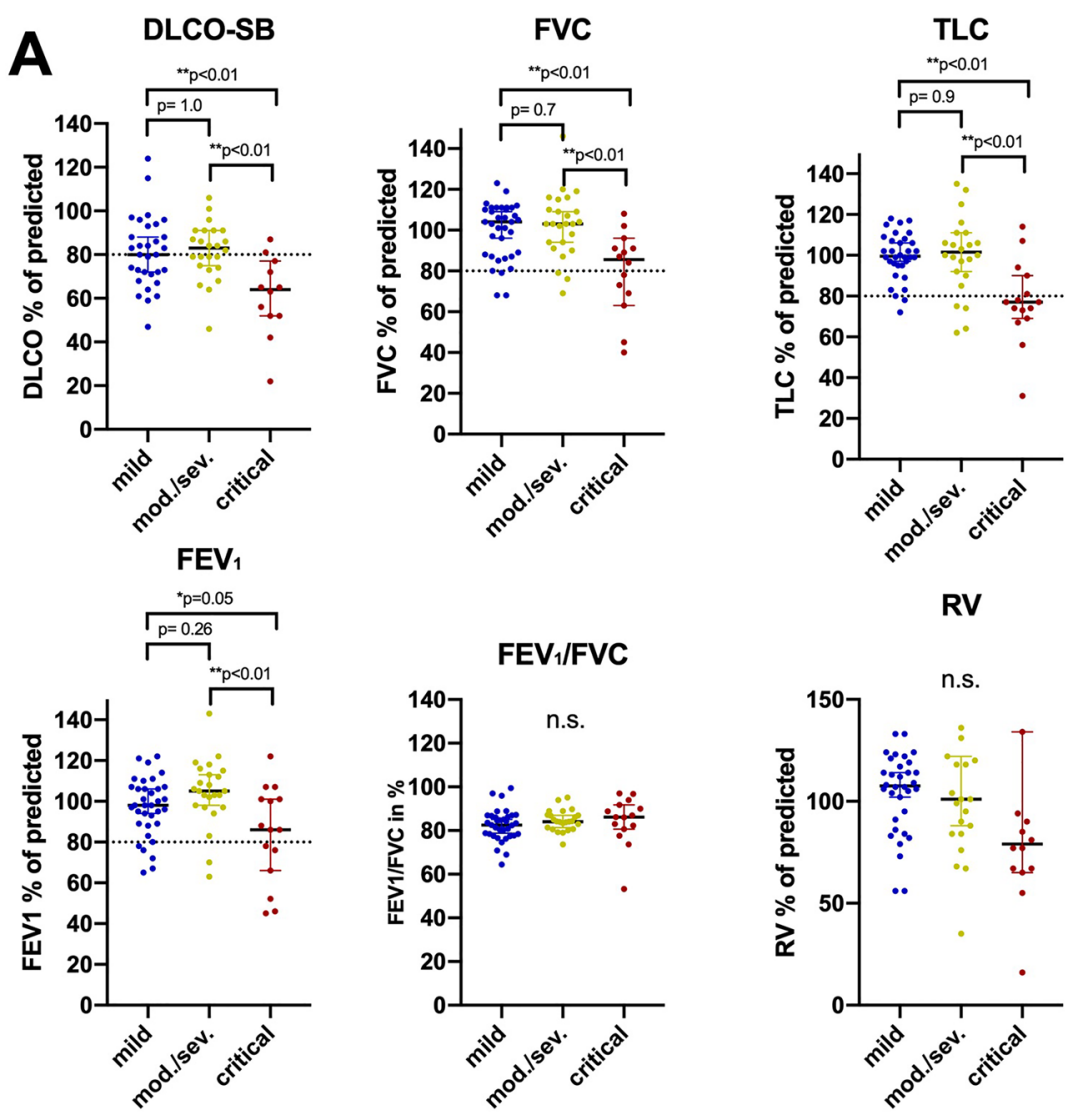

B paO2 at rest
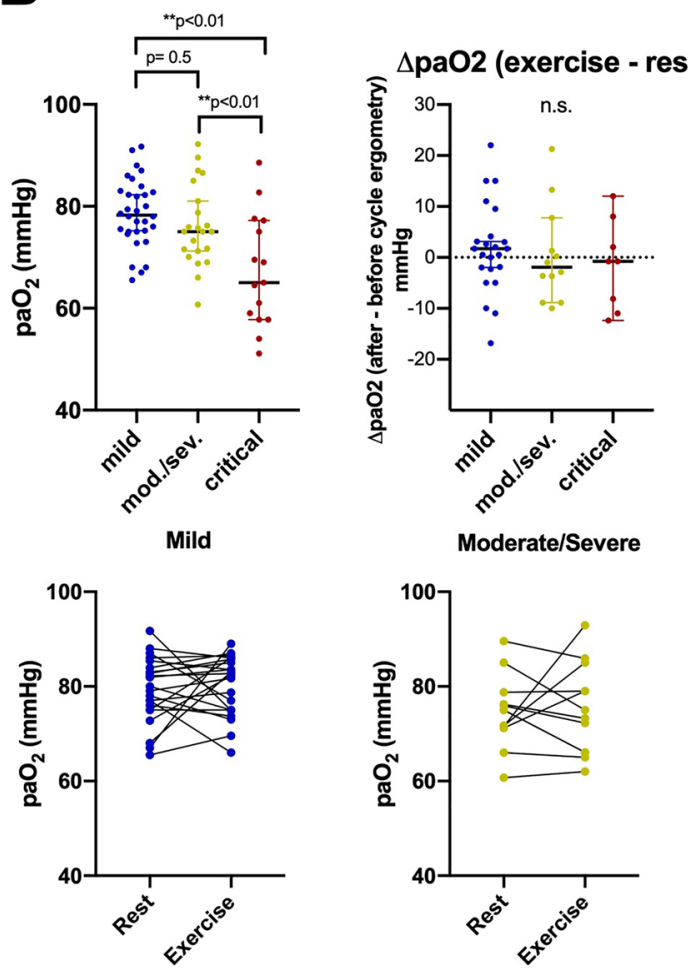

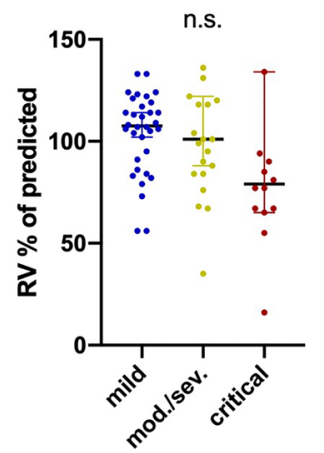

AaDO2 during exercise
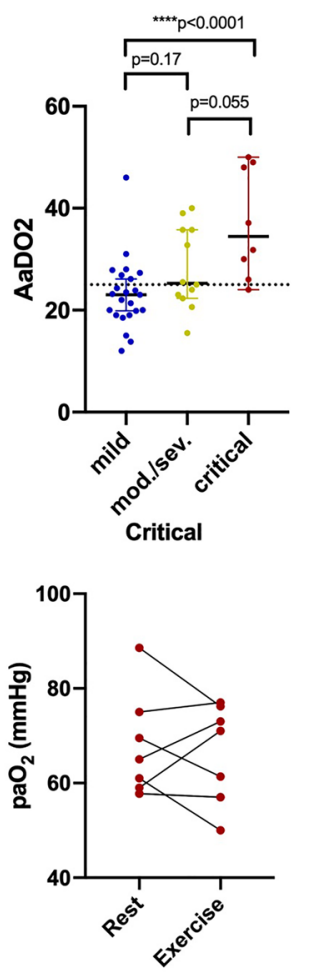
Table 3 (A) Univariate regression analysis; (B) multivariate regression analysis in the total cohort, (C) multivariate regression analysis in the hospitalized cohort (D) multivariate regression analysis in the non-critical cohort

\begin{tabular}{|c|c|c|c|c|c|c|c|c|}
\hline \multicolumn{9}{|l|}{ A } \\
\hline \multirow[t]{3}{*}{ All patients, $n=76$, univariate regression } & \multicolumn{2}{|l|}{ DLCOcSB } & \multicolumn{2}{|l|}{ TLC } & \multicolumn{2}{|l|}{$\mathrm{FVC}$} & \multicolumn{2}{|l|}{ FEV1 } \\
\hline & \multicolumn{2}{|l|}{ ULR } & \multicolumn{2}{|l|}{ ULR } & \multicolumn{2}{|l|}{ ULR } & \multicolumn{2}{|l|}{ ULR } \\
\hline & $p$ value & Coeff. $r$ & $p$ value & Coeff. $r$ & $p$ value & Coeff. $r$ & $p$ value & Coeff. $\mathrm{r}$ \\
\hline Mechanical ventilation $($ yes $=1$, no $=0$ ) & $<0.001 * *$ & -0.453 & $<0.001 * *$ & -0.488 & $<0.001 * *$ & $*-0.465$ & $0.002 *$ & -0.349 \\
\hline Duration of mechanical ventilation (days) & $0.002 * *$ & -0.378 & $<0.001 * *$ & -0.401 & $0.001 *$ & -0.39 & $0.020 *$ & -0.27 \\
\hline Duration of hospitalization (days) & $0.002 * *$ & -0.378 & $0.025^{*}$ & -0.266 & 0.015 & -0.287 & 0.141 & -0.175 \\
\hline $\begin{array}{l}\text { Oxygen insufflation necessary (yes }=1 \text {, } \\
\text { no=0) }\end{array}$ & $0.006 * *$ & 0.332 & $0.003 * *$ & -0.342 & $0.019 *$ & -0.727 & 0.312 & -0.119 \\
\hline Charlson Index & $0.001 *$ & -0.401 & 0.088 & -0.201 & 0.085 & -0.201 & 0.121 & -0.182 \\
\hline COPD $($ yes $=1$, no $=0)$ & $0.010 *$ & -0.313 & $0.006 * *$ & -0.319 & $0.007 *$ & -0.312 & $0.002 *$ & -0.355 \\
\hline Bronchial asthma (yes $=1$, no=0) & 0.191 & -0.162 & 0.158 & -0.167 & 0.372 & -0.116 & 0.106 & -0.189 \\
\hline Pack years & $0.005^{*}$ & -0.339 & 0.097 & -0.196 & $0.006 *$ & -0.319 & $0.001 *$ & -0.376 \\
\hline Type 2 diabetes (yes $=1$, no $=0$ ) & 0.802 & 0.031 & 0.905 & 0.014 & 0.756 & -0.037 & 0.703 & 0.045 \\
\hline Coronary artery disease $($ yes $=1$, no $=0$ ) & 0.244 & -0.144 & 0.414 & -0.097 & 0.11 & -0.188 & 0.223 & -0.143 \\
\hline Obesity $($ yes $=1$, no $=0)$ & $0.042 *$ & -0.25 & $0.003 * *$ & -0.348 & $<0.001 * *$ & $*-0.44$ & $0.001 *$ & -0.374 \\
\hline Immunosuppression $($ yes $=1$, no $=0$ ) & 0.09 & -0.209 & 0.742 & 0.039 & 0.979 & 0.003 & 0.392 & -0.101 \\
\hline SARS-CoV-2 IgG Antibody titer & $\mathbf{0 . 0 0 3} * *$ & -0.385 & $0.001 * *$ & -0.398 & $0.033^{*}$ & -0.269 & 0.331 & -0.115 \\
\hline Persistent symptoms & 0.454 & 0.092 & 0.45 & -0.088 & 0.214 & -0.144 & 0.295 & -0.12 \\
\hline Persistent respiratory symptoms & 0.076 & 0.205 & 0.937 & -0.009 & 0.438 & -0.09 & 0.985 & 0.002 \\
\hline \multicolumn{9}{|l|}{ B } \\
\hline \multirow[t]{3}{*}{ All patients, $n=76$, multivariate regression } & \multicolumn{2}{|l|}{ DLCOcSB } & \multicolumn{2}{|l|}{ TLC } & \multicolumn{2}{|l|}{ FVC } & \multicolumn{2}{|l|}{ FEV1 } \\
\hline & \multicolumn{2}{|l|}{ MLR } & \multicolumn{2}{|l|}{ MLR } & \multicolumn{2}{|l|}{ MLR } & \multicolumn{2}{|l|}{ MLR } \\
\hline & $p$ value & Coeff. beta & $p$ value & Coeff. beta & $p$ value & Coeff. beta & $p$ value & Coeff. beta \\
\hline Mechanical ventilation $($ yes $=1$, no $=0$ ) & $0.009 * *$ & -0.308 & $<0.001 *$ & -0.49 & $<0.001 * *$ & -0.506 & $0.001 * *$ & -0.352 \\
\hline Duration of mech. ventilation & 0.913 & 0.016 & 0.608 & -0.08 & 0.781 & -0.043 & 0.894 & -0.022 \\
\hline Duration of hospitalization (days) & 0.51 & 0.086 & 0.472 & -0.099 & 0.427 & 0.102 & 0.546 & 0.089 \\
\hline $\begin{array}{l}\text { Oxygen insufflation necessary }(\text { yes }=1, \\
\text { no }=0 \text { ) }\end{array}$ & 0.44 & 0.113 & 0.732 & 0.048 & 0.589 & 0.07 & 0.146 & 0.216 \\
\hline Charlson Index & 0.282 & 0.126 & 0.059 & 0.198 & 0.065 & -0.547 & 0.256 & -0.128 \\
\hline COPD $($ yes $=1$, no $=0)$ & $0.007 * *$ & -0.264 & $0.001 * *$ & -0.318 & $\mathbf{0 . 0 0 3}^{* *}$ & -0.296 & $0.004 * *$ & -0.312 \\
\hline SARS-CoV-2 IgG Antibodies & $0.014 *$ & -0.291 & 0.105 & -0.199 & 0.245 & -0.134 & 0.845 & -0.026 \\
\hline \multicolumn{9}{|l|}{$\mathrm{C}$} \\
\hline \multirow[t]{3}{*}{ Hospitalized, $n=41$, multivariate regression } & \multicolumn{2}{|l|}{ DLCOcSB } & \multicolumn{2}{|l|}{ TLC } & \multicolumn{2}{|l|}{ FVC } & FEV1 & \\
\hline & MLR & & MLR & & MLR & & MLR & \\
\hline & $p$ value & Coeff. beta & $p$ value & Coeff. beta & $p$ value & Coeff. beta & $p$ value & Coeff. beta \\
\hline Computertomography Score (0-25) & 0.026 & -0.323 & $0.006 *$ & -0.419 & $0.006 * *$ & -0.419 & 0.66 & -0.071 \\
\hline Mechanical ventilation $($ yes $=1$, no $=0$ ) & $0.003 * *$ & -0.425 & $0.033^{*}$ & -0.321 & 0.033* & -0.321 & $0.001 * *$ & -0.514 \\
\hline Duration of intubation in days & 0.431 & -0.145 & 0.083 & -0.245 & 0.083 & -0.245 & 0.9 & -0.025 \\
\hline $\begin{array}{l}\text { Oxygen insufflation necessary (yes }=1 \text {, } \\
\text { no=0) }\end{array}$ & 0.166 & -0.213 & 0.126 & -0.216 & 0.506 & -0.102 & 0.867 & 0.028 \\
\hline SARS-CoV-2 IgG Antibodies & $0.042 *$ & -0.3 & 0.313 & -0.153 & 0.761 & -0.047 & 0.94 & -12 \\
\hline
\end{tabular}


Table 3 (continued)

\begin{tabular}{|c|c|c|c|c|c|c|c|c|}
\hline \multirow{3}{*}{$\begin{array}{l}\text { Mild versus moderate/severe } \\
N=60\end{array}$} & \multicolumn{2}{|c|}{ DLCOcSB } & \multicolumn{2}{|l|}{ TLC } & \multicolumn{2}{|l|}{ FVC } & \multicolumn{2}{|l|}{ FEV1 } \\
\hline & \multicolumn{2}{|l|}{ MLR } & \multicolumn{2}{|l|}{ MLR } & \multicolumn{2}{|l|}{ MLR } & \multicolumn{2}{|l|}{ MLR } \\
\hline & $p$ value & Coeff. beta & $p$ value & Coeff. beta & $p$ value & Coeff. beta & $p$ value & Coeff. beta \\
\hline Duration of hospitalization in days & 0.857 & -0.022 & 0.059 & 0.241 & 0.067 & 0.231 & 0.819 & -0.028 \\
\hline $\begin{array}{l}\text { Oxygen insufflation necessary }(\text { yes }=1, \\
\text { no }=0 \text { ) }\end{array}$ & 0.651 & -0.054 & 0.818 & 0.03 & 0.591 & 0.068 & 0.599 & -0.064 \\
\hline Charlson Index & 0.771 & 0.041 & $0.026^{*}$ & -0.287 & 0.254 & -0.16 & 0.225 & 0.179 \\
\hline COPD $($ yes $=1$, no $=0)$ & $0.039 *$ & -0.251 & 0.407 & -0.116 & 0.03 & -0.281 & 0.047 & -0.233 \\
\hline Immunocompromised $($ yes $=1$, no $=0$ ) & $0.002 * *$ & -0.391 & 0.69 & 0.053 & 0.286 & -0.136 & $<0.001 *$ & -0.476 \\
\hline SARS-CoV-2 IgG Titer & $0.050 *$ & -0.237 & 0.72 & -0.047 & 0.974 & 0.004 & $0.039 *$ & -0.249 \\
\hline
\end{tabular}

p-values $<0.05$ were considered significant and marked in bold

displayed hyperventilation compared to asymptomatic patients $(n=35$, paCO2: $35.0 \pm 2.9$ vs. $38.3 \pm 6.5 \mathrm{mmHg}$, $p=0.019 ; \mathrm{paO} 2: 76.4 \pm 6.9$ vs $75.5 \pm 11.1, p=0.862)$.

A drop of paO2 $>3 \mathrm{mmHg}$ during exercise occurred in $15 \%$ asymptomatic $(n=2$ of 13$)$ and in $37 \%(n=11$ of 30 , Figure S1) of symptomatic (all COVID-19 related symptoms) patients.

\section{Discussion}

Here we report comprehensive pulmonary function evaluation of COVID-19 patients 4 months after acute illness in a broad cohort of patients ranging from mild to critical disease course including patients with persisting symptoms as well as patients who were asymptomatic at the time-point of follow--up.

We found impaired pulmonary function in more than half of the patients with a reduction in DLCO as the most frequent impairment (95\% of patients with impairment). Recent studies reported reduced DLCO, TLC, and FVC one to six months after COVID-19 disease in selected patients; however, so far, most studies focused on hospitalized cohorts [21-26].

Contrary to these studies, we included $46 \%(n=35)$ patients who had a mild disease course without hospitalization and also $33 \%(n=25)$ who reported no persistent COVID-19-related symptoms. Even though patients with critical disease had more severe PFI at mean, we also found PFI defined as values $<80 \%$ predicted in a proportion of patients who had mild and moderate/severe COVID-19.

Reduced DLCO and lung capacities after COVID-19 may indicate interstitial lung damage. Interstitial lung disease after COVID-19 disease is an important clinical manifestation in the convalescent phase. Recent studies report up to $62 \%$ of pulmonary interstitial fibrosis-like changes two months after critical disease courses [27, 28]. Similar to ARDS, long-term mechanical ventilation likely plays an important role in the development of interstitial fibrosis. Even though we found mechanical ventilation was a main risk factor for DLCO impairment in this study, a significant percentage of moderate to severe patients without the need for mechanical ventilation had decreased DLCO (40\%). As preexisting COPD was rare, this suggests non-critical COVID-19 pneumonia as likely cause in most patients. Additionally, impaired perfusion could play a role, as thromboembolism and alveolar capillary microthrombi have been described in COVID-19 [29].

Long-term follow-up has not been established yet. It is unclear whether impaired diffusion capacity will improve and which risk factors for persistence over more extended time courses exist. Interestingly in SARS, DLCOi $(<80 \%)$ was only observed in $12.7 \%$ of patients at 3 months after acute illness [30]. Early identification of restrictive disease and identification of risk factors might be essential for early treatment interventions, and primarily measurement of DLCO appears crucial in patients after COVID-19. TLC and FVC are less frequently impaired and thus secondary markers for identifying patients at risk. If progressive fibrosing interstitial lung disease after COVID-19 is confirmed by further diagnostic assessment, antifibrotic agents, e.g. Nintedanib might be a treatment option [31], that needs to be studied in RCTs.

To elucidate risk factors for pulmonary function impairment, we integrated clinical data during acute illness. As mentioned above, the most substantial risk factor for impaired DLCO, TLC, FVC, and FEV1 was mechanical ventilation, in line with findings from other recent cohorts [21, 22, 24, 32]. Interestingly, elevated SARS-CoV-2 Titer independently predicted impaired DLCO among all 
patients. Higher levels of antibody response have been described in critical versus non-critical COVID-19 disease [33]. Deeper analyses found that 2 weeks after presentation IgG antibodies of patients with critical disease compared to those with moderate disease showed functional characteristics likely to induce monocyte/macrophage activation [34]. Thus antibody responses have been suggested to play a role in an exaggerated inflammatory response and immunopathology [35], with possibly detrimental effects on lung tissue. These mechanisms might explain consecutive diffusion impairment in our study.

Including only the inpatients with CT scan available during acute illness $(n=41)$, an additional negative predictor for DLCO impairment was increased initial CT score (MLR: $p=0.049$ ).

To further investigate gas exchange after COVID-19, we implemented steady-state cycle ergometry with blood gas analysis in eligible patients. Of note, a decrease of capillary $\mathrm{paO}_{2}>3 \mathrm{mmHg}$ or increased $\mathrm{AaDO} 2>25 \mathrm{mmHg}$ after cycle ergometry occurred after mild disease courses ( 8 of $23,35 \%$ and 7 of $23,30 \%$ ), revealing gas exchange abnormalities even in some patients who did not require hospitalization. Rinaldo et al. [36] recently performed cardiopulmonary exercise testing (CPET) with maximal exercise in 75 COVID-19 survivors three months after discharge. The researchers mainly reported impaired exercise capacity probably caused by muscular deconditioning in 41 patients (54.7\%) compared to 34 patients with normal exercise capacity while mean $\mathrm{AaDO} 2$ was 26 in both groups.

Also, among mild and moderate/severe patients $(n=60)$, pulmonary function impairment did not depend on the duration of hospitalization $(p=0.857)$ and oxygen insufflation $(p=0.651)$. These findings might indicate the presence of undiagnosed viral pneumonia and subsequent interstitial lung damage or perfusion abnormalities after mild disease courses.

Furthermore, our post-COVID-19 patients underwent a detailed pulmonologic evaluation. Postviral bronchial hyperreactivity syndrome is common after respiratory tract viral infections [37]; however, its prevalence after COVID-19 is unclear. Here, we investigated bronchial hyperresponsiveness only in patients with normal baseline lung function but persisting respiratory symptoms. We report 3 out of 8 challenged patients who had bronchial hyperresponsiveness after COVID-19, suggesting this may also be a complication of COVID-19. In total, only in 3.9\% patients bronchial hyperresponsiveness was confirmed, indicating a minor role of prior postulated postviral bronchial hyperreactivity $[37,38]$. The main symptoms were shortness of breath and persistent cough. All patients with bronchial hyperresponsiveness were treated with Beclometasone/Formoterol $100 \mu \mathrm{g} / 6 \mu \mathrm{g} 2-0-2$ for $6-8$ weeks and symptoms resolved in all patients within three months.
As expected, COPD was associated with impaired pulmonary function in this cohort. While these patients have preexisting reduced pulmonary function due to COPD, it is also a known risk factor for all-cause mortality and progression to severe disease in COVID-19 patients [39].

Moreover, smoking and pack-years are negatively correlated with lung function decline in UR. Pathophysiological mechanisms of smoking may lead to declined lung function before infection or contribute to worsening lung condition due to higher vulnerability.

At 4 months after acute illness, persistent COVID-19 specific symptoms occurred in all subgroups $(n=52 ; 68.4 \%)$, but patients with mild disease course were more frequently free of symptoms than the other groups. Interestingly, we found no significant correlation between persisting symptoms and impaired pulmonary function, indicating that other mechanisms may play an important role in the reported symptoms. Diverse mechanisms for long-/post-COVID symptoms have been proposed, but the exact pathophysiology remains elusive so far [40].

Contrary to other studies, we have also included patients with mild disease course and patients who did not report symptoms at follow-up and thus analyzed the influence of disease severity and persistent symptoms on PFI. This approach revealed that PFI might also be present after mild COVID-19, and such patients should also be included in future studies.

However, it should be noted that this was not a population-based study. At the time of presentation in our aftercare unit 23 patients $(65.7 \%)$ had persistent symptoms, whereas 12 patients (34.3\%) negated persistent COVID-19 related symptoms. Asymptomatic patients $(n=12)$ were referred or presented themselves due to intrinsic or scientific interest after announcing the post-COVID-19 aftercare offer after the first COVID-19 wave. Nevertheless, we assume that negative effects on lung function are less frequent in patients who do not present in an aftercare unit after mild disease courses. Potential selection bias might be present in the mild disease course group. Thus, the frequencies of symptomatic versus asymptomatic follow-up patients are not representative for an overall post-COVID-population. Additionally, analyses are limited due to moderate sample size, and symptoms were analyzed only qualitatively. As PFT values before the disease were not available for the majority of the patients, mild preexisting abnormalities cannot be excluded. Furthermore, in this study, we performed steady-state exercise and not complete cardiopulmonary exercise testing (CPET). Interestingly, in mild disease, paCO2 was at mean $3.3 \mathrm{mmHg}$ lower in symptomatic patients than in asymptomatic patients, but clinical significance of this small difference is currently unclear.

According to our data and recent publications, we recommend pulmonary aftercare to patients after severe and 
critical COVID-19 disease courses [11, 23, 32, 39, 41]. In particular, patients with initial elevated CT values and after mechanical ventilation should be considered for referral. In addition, patients with preexisting lung conditions (COPD), former or active smokers, and patients with persistent pulmonary symptoms (cough, dyspnea, fatigue) might profit from pulmonary assessment. If after diagnostic evaluation (lung function, CT scan, echocardiography and others) persistent symptoms remain unclear, CPET might be an option to discriminate deconditioning from severe other causes [36].

In sum, we describe that PFI may be present in symptomatic and asymptomatic post-COVID-19 patients but is most frequent in those who had severe acute illness. Interestingly, a decrease of $\mathrm{paO} 2$ upon exercise was found in $1 / 5$ of the mild disease patients, most of whom had borderline-mild DLCO impairment at rest. Thus, exercise testing should be included in post-COVID-19 evaluation as it may reveal subtle gas exchange abnormalities, which could be responsible for some of the persisting symptoms.

Supplementary Information The online version contains supplementary material available at https://doi.org/10.1007/s15010-021-01669-8.

Author contributions KM: perception of the study, treatment of patients, analysis and illustration, drafting of the manuscript. NK: perception of the study, treatment of patients. DM and TV: data curation, analysis and illustration, drafting of the manuscript. JW: statistical analysis. JD: radiologic analysis and CT scoring. AO and MM: virologic analyses, CORKUM registry platform data. JCH: CORKUM registry platform and data. PM, CM, EK, MB, SM and JB: treatment of patients. AG, DM and KM: additional analyses in the revision process. All authors: revision of the manuscript and accordance for submission.

Funding Open Access funding enabled and organized by Projekt DEAL.

\section{Declarations}

Conflict of interest On behalf of all authors, the corresponding author states that there is no conflict of interest.

Ethical standards The study was approved by the ethics committee of the LMU (project number 20-454) and has been performed in accordance with the ethical standards laid down in the 1964 Declaration of Helsinki and its later amendments.

Open Access This article is licensed under a Creative Commons Attribution 4.0 International License, which permits use, sharing, adaptation, distribution and reproduction in any medium or format, as long as you give appropriate credit to the original author(s) and the source, provide a link to the Creative Commons licence, and indicate if changes were made. The images or other third party material in this article are included in the article's Creative Commons licence, unless indicated otherwise in a credit line to the material. If material is not included in the article's Creative Commons licence and your intended use is not permitted by statutory regulation or exceeds the permitted use, you will need to obtain permission directly from the copyright holder. To view a copy of this licence, visit http://creativecommons.org/licenses/by/4.0/.

\section{References}

1. Li Q, et al. Early transmission dynamics in Wuhan, China, of novel coronavirus-infected pneumonia. N Engl J Med. 2020;382:1199-207.

2. Chen N, et al. Epidemiological and clinical characteristics of 99 cases of 2019 novel coronavirus pneumonia in Wuhan, China: a descriptive study. Lancet. 2020;395:507-13.

3. Huang C, et al. Clinical features of patients infected with 2019 novel coronavirus in Wuhan, China. Lancet. 2020;395:497-506.

4. Wang D, et al. Clinical characteristics of 138 hospitalized patients with 2019 novel coronavirus-infected pneumonia in Wuhan, China. JAMA. 2020;323:1061-9.

5. Jakob CEM, et al. First results of the "Lean European Open Survey on SARS-CoV-2-Infected Patients (LEOSS).” Infection. 2021;49:63-73.

6. Needham DM, et al. Improving long-term outcomes after discharge from intensive care unit: report from a stakeholders' conference. Crit Care Med. 2012;40:502-9.

7. Bieber S, et al. Left and right ventricular dysfunction in patients with COVID-19-associated myocardial injury. Infection. 2021;49:491-500.

8. Spiro JE, et al. Secondary tension pneumothorax in a COVID-19 pneumonia patient: a case report. Infection. 2020;48:941-4.

9. Weber S, et al. Severe liver failure during SARS-CoV-2 infection. Gut. 2020;69:1365-7.

10. Tan BK, et al. Arterial and venous thromboembolism in COVID19: a study-level meta-analysis. Thorax. 2021. https://doi.org/10. 1136/thoraxjnl-2020-215383.

11. Huang $\mathrm{C}$, et al. 6-month consequences of COVID-19 in patients discharged from hospital: a cohort study. Lancet. 2021;397:220-32.

12. Quanjer PH, et al. Lung volumes and forced ventilatory flows. Report Working Party Standardization of Lung Function Tests, European Community for Steel and Coal. Official Statement of the European Respiratory Society. Eur Respir J. 1993;6:5-40.

13. Quanjer PH, et al. Multi-ethnic reference values for spirometry for the 3-95-yr age range: the global lung function 2012 equations. Eur Respir J. 2012;40:1324-43.

14. Coates AL, et al. ERS technical standard on bronchial challenge testing: general considerations and performance of methacholine challenge tests. Eur Respir J. 2017;49:1601526.

15. Munker D, et al. Dynamics of SARS-CoV-2 shedding in the respiratory depends on the severity of disease in COVID-19 patients. Eur Respir J. 2021. https://doi.org/10.1183/13993003. 02724-2020.

16. Charlson ME, et al. A new method of classifying prognostic comorbidity in longitudinal studies: development and validation. J Chronic Dis. 1987;40:373-83.

17. Antonio GE, et al. Thin-section CT in patients with severe acute respiratory syndrome following hospital discharge: preliminary experience. Radiology. 2003;228:810-5.

18. Chung M, et al. CT imaging features of 2019 novel coronavirus (2019-nCoV). Radiology. 2020;295:202-7.

19. Dai H, et al. High-resolution chest CT features and clinical characteristics of patients infected with COVID-19 in Jiangsu, China. Int J Infect Dis. 2020;95:106-12.

20. Muenchhoff M, et al. Multicentre comparison of quantitative PCR-based assays to detect SARS-CoV-2, Germany, March 2020. Euro Surveill. 2020. https://doi.org/10.2807/1560-7917. ES.2020.25.24.2001057.

21. Huang Y, et al. Impact of coronavirus disease 2019 on pulmonary function in early convalescence phase. Respir Res. 2020;21:163. 
22. You J, et al. Anormal pulmonary function and residual CT abnormalities in rehabilitating COVID-19 patients after discharge. J Infect. 2020;81:e150-2.

23. Anastasio F, et al. Medium-term impact of COVID-19 on pulmonary function, functional capacity and quality of life. Eur Respir J. 2021. https://doi.org/10.1183/13993003.04015-2020.

24. Torres-Castro R, et al. Respiratory function in patients postinfection by COVID-19: a systematic review and meta-analysis. Pulmonology. 2020. https://doi.org/10.1016/j.pulmoe.2020.10. 013.

25. Fumagalli A, et al. Pulmonary function in patients surviving to COVID-19 pneumonia. Infection. 2021;49:153-7.

26. Fortini A, et al. COVID-19: persistence of symptoms and lung alterations after 3-6 months from hospital discharge. Infection. 2021. https://doi.org/10.1007/s15010-021-01638-1.

27. Zou H, Li SQ. Pulmonary fibrosis in critically ill patients with novel coronavirus pneumonia during the convalescent stage and a proposal for early intervention. Acta Pharmacol Sin. 2020. https:// doi.org/10.1038/s41401-020-00566-4.

28. Liu Q, et al. Gross examination report of a COVID-19 death autopsy. Fa Yi Xue Za Zhi. 2020;36:21-3.

29. Ackermann M, et al. Pulmonary vascular endothelialitis, thrombosis, and angiogenesis in Covid-19. N Engl J Med. 2020;383:120-8.

30. Hui DS, et al. Impact of severe acute respiratory syndrome (SARS) on pulmonary function, functional capacity and quality of life in a cohort of survivors. Thorax. 2005;60:401-9.

31. Wells AU, et al. Nintedanib in patients with progressive fibrosing interstitial lung diseases-subgroup analyses by interstitial lung disease diagnosis in the INBUILD trial: a randomised, doubleblind, placebo-controlled, parallel-group trial. Lancet Respir Med. 2020;8:453-60.
32. Guler SA, et al. Pulmonary function and radiological features four months after COVID-19: first results from the national prospective observational Swiss COVID-19 lung study. Eur Respir J. 2021. https://doi.org/10.1183/13993003.03690-2020.

33. Qu J, et al. Profile of immunoglobulin G and IgM antibodies against severe acute respiratory syndrome coronavirus 2 (SARSCoV-2). Clin Infect Dis. 2020;71:2255-8.

34. Zohar T, et al. Compromised humoral functional evolution tracks with SARS-CoV-2 mortality. Cell. 2020;183:1508-1519 e12.

35. Seddiki N, French M. COVID-19 and HIV-associated immune reconstitution inflammatory syndrome: emergence of pathogenspecific immune responses adding fuel to the fire. Front Immunol. 2021;12:649567.

36. Rinaldo RF, et al. Deconditioning as main mechanism of impaired exercise response in COVID-19 survivors. Eur Respir J. 2021. https://doi.org/10.1183/13993003.00870-2021.

37. Ostransky D, Blais FX. Postviral bronchial hyperreactivity syndrome: recognizing asthma's great mimic. J Am Osteopath Assoc. 1991;91(5): 465-8, 471-5.

38. Folkerts $\mathrm{G}$, et al. Virus-induced airway hyperresponsiveness and asthma. Am J Respir Crit Care Med. 1998;157:1708-20.

39. Lee SC, et al. Impact of COPD on COVID-19 prognosis: A nationwide population-based study in South Korea. Sci Rep. 2021;11:3735

40. Venkatesan P. NICE guideline on long COVID. Lancet Respir Med. 2021;9:129.

41. Zhao YM, et al. Follow-up study of the pulmonary function and related physiological characteristics of COVID-19 survivors three months after recovery. EClinicalMedicine. 2020;25:100463.

\section{Authors and Affiliations}

Dieter Munker ${ }^{1,2} \cdot$ Tobias Veit $^{1,2} \cdot$ Jürgen Barton ${ }^{1,2} \cdot$ Pontus Mertsch $^{1,2} \cdot$ Carlo Mümmler $^{1,2} \cdot$ Andreas Osterman $^{3,4}$. Elham Khatamzas ${ }^{5}$. Michaela Barnikel ${ }^{1,2}$. Johannes C. Hellmuth ${ }^{5,6}$. Maximilian Münchhoff ${ }^{3,4,6}$. Julia Walter ${ }^{1,2}$. Alessandro Ghiani ${ }^{7} \cdot$ Stefan Munker ${ }^{8} \cdot$ Julien Dinkel $^{9} \cdot$ Jürgen Behr ${ }^{1,2} \cdot$ Nikolaus Kneidinger $^{1,2} \cdot$ Katrin Milger $^{1,2}(\mathbb{C}$

1 Department of Medicine V, University Hospital, LMU Munich, Marchioninistr. 15, 81377 Munich, Germany

2 Comprehensive Pneumology Center Munich (CPC-M), Helmholtz Center and LMU Munich, Member of the German Center for Lung Research (DZL), Munich, Germany

3 Max von Pettenkofer Institute and Gene Center, Virology, National Reference Center for Retroviruses, Ludwig Maximilian University (LMU) of Munich, Munich, Germany

4 German Center for Infection Research (DZIF), Partner Site Munich, Munich, Germany

5 Department of Medicine III, University Hospital, LMU Munich, Munich, Germany
6 COVID-19 Registry of the LMU Munich (CORKUM), University Hospital, Ludwig-Maximilians University Munich, Munich, Germany

7 Department of Pulmonology and Respiratory Medicine, Schillerhoehe Lung Clinic (affiliated to the Robert-Bosch-Hospital GmbH, Stuttgart), Solitudestrasse 18, 70839 Gerlingen, Germany

8 Department of Medicine II, University Hospital, LMU Munich, Munich, Germany

9 Department of Radiology, University Hospital, LMU Munich, Munich, Germany 